\title{
OPINIONES SOBRE LAS PRÁCTICAS ANTICONCEPTIVAS EN ESTUDIANTES DE UNA FACULTAD DE HUMANIDADES EN SANTA MARTA
}

\section{ATTITUDES ABOUT OPINIONS AND PRACTICES IN STUDENTS OF BIRTH CONTROL A FACULTY OF HUMANITIES IN SANTA MARTA}

\author{
TITULO CORTO: OPINIONES SOBRE LAS PRÁCTICAS ANTICONCEPTIVAS EN \\ ESTUDIANTES
}

Ubaldo Rodríguez-De Ávilaㄹ, Johanna Bocanegra-Sandoval², Edilberto Maestre-Oñate ${ }^{3}$, Sergio Lozano-González ${ }^{4}$

Recibido en febrero 04 de 2016

Aceptado en abril 11 de 2016

\section{RESUMEN}

En el presente estudio se buscó determinar las opiniones sobre los métodos anticonceptivos y la relación existente entre la variable de estudio, el programa académico y el género en estudiantes de la facultad de humanidades de la Universidad del Magdalena. Mediante el cual se brindan datos actualizados sobre las conductas sexuales de riesgo de los estudiantes, los cuales pueden ser útiles para la prevención de esta problemática, por ello se realizó un estudio descriptivo-correlacional, utilizando un muestreo intencional, con una participación de 120 estudiantes. Los hallazgos permitieron concluir que los estudiantes plantean opiniones positivas y mayor uso sobre el preservativo y las píldoras; asimismo poseen opiniones negativas de la abstinencia, el dispositivo intrauterino y los implantes. Por otro lado, no se encontró relación entre el género y las opiniones sobre los métodos anticonceptivos, igualmente la opinión de los estudiantes no difiere según el programa.

Palabras clave: Anticonceptivos; Ciencias humanas; Relaciones sexuales

\section{ABSTRACT}

In the present study views on contraception and the relationship between the variable gender study and students of the faculty of humanities at the University of Magdalena are determined. This article would provide data on sexual risk behaviors of students, which can be useful for the prevention of this problem, thus a descriptive correlational study was conducted using purposive sampling, with a participation of 120 students. The findings support the conclusion that students pose positive reviews and increased use of condoms and pills also have negative opinions 
of abstinence, intrauterine devices and implants. On the other hand, there is no relationship between gender and views on contraception, also the opinion of students does not differ according to the program.

Keywords: contraceptives; human sciences; sexual intercourse

\section{INTRODUCCIÓN}

La salud sexual y reproductiva constituye en uno de los objetivos de los gobiernos y organizaciones a nivel mundial, pues considera que existen diversas repercusiones negativas asociadas a conductas que se convierten en factor de riesgo para la vida sexual de los sujetos. En el mundo se presentan alarmantes cifras que demuestran la vulnerabilidad de los adultos jóvenes en temas relacionados con la sexualidad, ya que cada año se registran en todo el mundo más de 340 millones de casos nuevos de enfermedades de transmisión sexual, evidenciando que 4,1 millones de casos nuevos de infección por VIH a nivel mundial, se dan entre jóvenes de 15 a 24 años de edad. Asimismo, además de la alta prevalencia de ETS, preocupa también las altas tasas de embarazos precoces y las diversas complicaciones que conllevan los embarazos a temprana edad ${ }^{1}$.

Se ha estimado que las principales causas de mortalidad en toda América, para el grupo entre 15 y 24 años, son las causas externas, entre las cuales se incluyen los accidentes, homicidios, suicidios y otras, seguidas por causas derivadas de complicaciones en el embarazo, parto y puerperio a temprana edad. Para el caso de las enfermedades transmisibles, en específico las ETS, dentro de las que se incluye VIH/sida, estas afectan a uno de cada 20 jóvenes por año, apareciendo entre las primeras cinco causas de muerte en personas entre los 15 y 24 años ${ }^{2}$.

En Colombia, la prevalencia de ETS tales como sífilis, infección gonocóccica, trichomoniasis urogenital, hepatitis $\mathrm{B}$ y herpes genital se presenta en altas tasas. Igualmente, el VIH ha aumentado su incidencia en mujeres y hombres con prácticas homosexuales ${ }^{3,4}$. El papilomavirus o virus del papiloma humano (VPH) se constituye en un factor de riesgo para el desarrollo del cáncer de cuello uterino, el cual presenta elevados niveles en relación con otros países, al convertirse en la primera causa de muerte en mujeres que se encuentran entre los 30 y 59 años, siendo la segunda causa de mortalidad por neoplasias 5 . Aunque las muertes asociadas al cáncer uterino se encuentran después de los 30 años, es importante destacar, que de acuerdo a la tendencia a iniciar a más temprana edad las relaciones sexuales, esta patología podría afectar cada vez a mujeres más jóvenes.

La Conferencia Internacional sobre Población y Desarrollo de Naciones Unidas (CIPD) de El Cairo (1994) se expresó la definición de salud sexual y reproductiva como un "Estado de bienestar físico, psíquico, emocional y social en relación a la sexualidad". Es así como la salud reproductiva tiene en cuenta la "capacidad de disfrutar de una vida sexual satisfactoria, sin riesgos, procrear, con libertad para elegir hacerlo o no hacerlo, cuándo y con qué frecuencia" ${ }^{\circ}$. Esta última condición lleva implícito el derecho que tienen los individuos de recibir información sobre los métodos anticonceptivos, sobre la elección del método de su conveniencia e inclinación.

Es importante precisar que los anticonceptivos o métodos de planificación familiar, son los mecanismos empleados por las personas para controlar la natalidad y para evitar enfermedades de transmisión sexual, las cuales son consideradas como un problema de salud pública $^{3,7-11}$. Las conductas de riesgo de salud sexual que afectan a la población (ETS, embarazos a temprana edad y las consecuencias para la salud derivados de estos), se pueden contrarrestar con la prevención, como herramienta imprescindible en la disminución de los altos niveles de esta problemática. Por esta razón se ha buscado la determinación de las opiniones de la población universitaria sobre las prácticas anticonceptivas.

El preservativo es considerado como herramienta para la prevención de ETS (incluyendo el VIH) y de embarazos no planeados, por esta razón se han estudiado las opiniones asumida por las personas sobre este. Los hombres reportan mayor uso del preservativo en relación a las mujeres, sin embargo, se observa una tendencia a no aplicarlos de forma regular, lo que pone de manifiesto el riesgo al que se ven expuestos ${ }^{12-14}$. Asimismo, la falta de uso de 
métodos de anticoncepción, incluyendo el preservativo, se relaciona con el poco conocimiento que los jóvenes tienen sobre estos, por creencias erróneas (autopercepción de bajo riesgo, sentido de invulnerabilidad) y ausencia de habilidades necesarias para su correcto uso $0^{8,12,13,15-18}$. Por otro lado, el ritmo, la esterilización femenina y la inyección se encuentran entre los métodos anticonceptivos más utilizados tanto por hombres como por mujeres ${ }^{17-19}$, sin embargo, vale la pena resaltar que estos métodos son poco eficaces para la prevención de ETS.

Teniendo como referente el riesgo que representa para la salud los embarazos a temprana edad y las enfermedades de transmisión sexual, se hizo importante llevar a cabo esta investigación con el objetivo de determinar las opiniones sobre los métodos anticonceptivos en estudiantes de la facultad de humanidades de la Universidad del Magdalena.

\section{MATERIALES Y MÉTODOS}

Esta investigación es cuantitativa, con diseño descriptivo correlacional, lo cual permite describir las variables como son observadas, a su vez, evidencia las relaciones existentes entre dos o más variables, logrando establecer la relación existente entre las opiniones, el programa académico (cine y audiovisuales, antropología y derecho) y el género (masculino y femenino); enmarcado dentro del paradigma empírico-analítico, el cual analiza de forma particular una variable, basándose en información empírica objetiva, es decir, "cosas o aspectos que se pueden medir, los cuales por su estructura siempre arrojan números como resultado" ${ }^{20}$.

\section{Participantes}

La población estuvo conformada por estudiantes de la facultad de Humanidades de la Universidad del Magdalena del periodo académico 2015-II. La muestra fue de 121 estudiantes escogidos de forma intencional por conveniencia considerando como criterios de inclusión ser estudiante matriculado en los programas de cine y audiovisuales, derecho y antropología, acorde a la tabla 1.

Lo anterior indica que 121 estudiantes participaron de la presente investigación de los cuales 73 eran mujeres lo cual corresponde a un $60,3 \%$ de la muestra, por su parte el $39,7 \%$ restante eran hombres con un total de 48 participantes.

Tabla 1. Descripción de la muestra

\begin{tabular}{|c|c|c|c|c|c|c|}
\hline \multirow{2}{*}{} & \multicolumn{2}{|c|}{ Mujeres } & \multicolumn{2}{c|}{ Hombres } & \multicolumn{2}{c|}{ Total } \\
\cline { 2 - 6 } & Frec. & $\%$ & Frec. & $\%$ & Frec. & $\%$ \\
\hline Cine y audiovisuales & 26 & $21,5 \%$ & 19 & $15,7 \%$ & 45 & $37,2 \%$ \\
\hline Derecho & 30 & $24,8 \%$ & 15 & $12,4 \%$ & 45 & $37,2 \%$ \\
\hline Antropología & 17 & $14 \%$ & 14 & $11,6 \%$ & 31 & $25,6 \%$ \\
\hline Total & 73 & $60,3 \%$ & 48 & $39,7 \%$ & 121 & $100,0 \%$ \\
\hline
\end{tabular}

Fuente: elaboración propia.

\section{Instrumento}

En el desarrollo de la investigación y como método de recolección de datos, se utilizó la Escala de Evaluación de Actitudes y Opiniones de Practicas Anticonceptivas en 14 países de Latinoamérica, diseñado y validado en el año 2010, el cuestionario es de fácil aplicación, comprende 5 opciones de respuesta $(1=$ totalmente en desacuerdo a $5=$ totalmente de acuerdo), para evaluar actitudes y opiniones hacia las practicas anticonceptivas, que incluye preguntas que evalúan las dos variables en mención ${ }^{21}$. El instrumento incluye 9 preguntas que evalúan actitudes en torno a prácticas anticonceptivas; donde se exploran específicamente las actitudes hacia un único constructo teórico. Su método de evaluación es ordinal $(1=$ totalmente en desacuerdo a $5=$ totalmente de acuerdo). La línea de corte para establecer la actitud global desfavorable o favorable hacia el uso de los métodos anticonceptivos fue de 28 (mediana) con una amplitud de clase (rango) de 9 a 45 puntos $^{21}$. 
Para evaluar las opiniones, el instrumento incluyó 12 preguntas, también se utilizó una escala ordinal tipo Likert de 5 opciones $(1=$ muy mala a $5=$ muy buena). La línea de corte para establecer la opinión global mala o buena hacia el uso de los métodos anticonceptivos fue de 36 (mediana) con una amplitud de clase (rango) de 12 a 60 puntos ${ }^{21}$.

Al momento de comprobar la validez de la escala, se halló un Alfa de Cronbach de 0.875 , lo cual permite afirmar que estadísticamente el instrumento posee adecuada consistencia interna, siendo altamente confiable y válido para ser usado en nuestra población.

\section{Procedimiento}

La investigación inició con la selección del tema a investigar, el cual se encuentra mediado por la motivación de los investigadores, y por una situación problemática. Luego, para formular y delimitar el problema, se efectuó un proceso de revisión bibliográfica, de fuentes primarias y secundarias, sobre la problemática que se plantea abordar. Este proceso se desarrolló simultáneamente a la revisión de la literatura y los estudios existentes, dando como resultado el estado del arte y el marco teórico.

Para alcanzar el objetivo de la investigación, inicialmente se solicitó la firma del consentimiento informado y posteriormente se evaluó las actitudes y opiniones hacia las prácticas anticonceptivas en estudiantes de la facultad de humanidades de la Universidad del Magdalena, la cual incluye a estudiantes de derecho, antropología, cine y audiovisuales.
El análisis de los datos obtenidos se llevó a cabo mediante el programa estadístico SPSS en su versión 18.0, en el cual se realizará un estudio descriptivo de las frecuencias, comprobación del Alfa de Cronbach y para corroborar las hipótesis se utilizó el estadístico de correlacion chi cuadrado. Finalmente, de acuerdo a los resultados se efectuó un proceso de discusión de los datos obtenidos, los cuales permitieron construir las conclusiones pertinentes. Este estudio se tuvo en cuenta las normas científicas, técnicas y administrativas para la investigación con seres humanos.

\section{Declaración de aspectos éticos.}

El presente estudio a pesar de no presentar riesgos para la salud de los participantes, tuvo en cuenta lo estipulado por en la resolución 8430 de 1993 del Ministerio de Salud, donde se establecen las normas científicas, técnicas y administrativas para la investigación en salud mental; así como también, lo dictaminado en la Ley 1090 de 2006 por el cual se establece y reglamenta el Código Deontológico y Ético del Psicólogo ${ }^{22,23}$.

\section{RESULTADOS}

El estudio describe la relación entre el género, el programa y las opiniones sobre los métodos anticonceptivos en estudiantes universitarios, para dar respuesta a los objetivos se procederá inicialmente a la descripción de dos categorías: Relación entre las opiniones acordes al sexo de la persona participante posteriormente la relación entre las opiniones y el programa.

Tabla 2. Sexo y uso de métodos anticonceptivos

\begin{tabular}{|c|c|c|c|c|c|c|}
\hline \multirow{2}{*}{ Sexualmente activo } & \multicolumn{2}{|c|}{ Mujeres } & \multicolumn{2}{c|}{ Hombres } & \multicolumn{2}{c|}{ Total } \\
\cline { 2 - 7 } & Frec. & $\%$ & Frec. & $\%$ & Frec. & $\%$ \\
\hline Si & 56 & $46,3 \%$ & 42 & $34,7 \%$ & 98 & $81 \%$ \\
\hline No & 17 & $14 \%$ & 6 & $5 \%$ & 23 & $19 \%$ \\
\hline Total & 73 & $60,3 \%$ & 48 & $39,7 \%$ & 121 & $100 \%$ \\
\hline \multirow{2}{*}{$\begin{array}{c}\text { Uso de métodos } \\
\text { anticonceptivos }\end{array}$} & \multicolumn{2}{|c|}{ Femenino } & \multicolumn{2}{|c|}{ Masculino } & \multicolumn{2}{c|}{ Total } \\
\cline { 2 - 8 } & Frec. & $\%$ & Frec. & Frec. & $\%$ & Frec. \\
\hline Si & 56 & $46,3 \%$ & 39 & $32,2 \%$ & 95 & $78,5 \%$ \\
\hline No & 17 & $14 \%$ & 9 & $7,4 \%$ & 26 & $21,4 \%$ \\
\hline Total & 73 & $60,3 \%$ & 48 & $39,7 \%$ & 121 & $100 \%$ \\
\hline
\end{tabular}


Continuación Tabla 2.

\begin{tabular}{|c|c|c|c|c|c|c|}
\hline \multirow{2}{*}{$\begin{array}{c}\text { Método } \\
\text { anticonceptivo } \\
\text { utilizado }\end{array}$} & \multicolumn{2}{|c|}{ Femenino } & \multicolumn{2}{c|}{ Masculino } & \multicolumn{2}{c|}{ Total } \\
\cline { 2 - 7 } & Frec. & $\%$ & Frec. & $\%$ & Frec. & $\%$ \\
\hline Calendario o ritmo & 7 & $5,8 \%$ & 4 & $3,3 \%$ & 11 & $9,1 \%$ \\
\hline Condón & 26 & $21,5 \%$ & 30 & $24,8 \%$ & 56 & $46,3 \%$ \\
\hline Inyección & 5 & $4,1 \%$ & 1 & $0,8 \%$ & 6 & $5 \%$ \\
\hline Pastillas & 17 & $14 \%$ & 3 & $2,5 \%$ & 20 & $16,5 \%$ \\
\hline Ninguno & 18 & $14,9 \%$ & 10 & $8,3 \%$ & 28 & $23,1 \%$ \\
\hline Total & 73 & $60,3 \%$ & 48 & $39,7 \%$ & 121 & $100 \%$ \\
\hline
\end{tabular}

Fuente: elaboración propia.

Tal como se observa en la tabla 2, al analizar el uso de métodos anticonceptivos de los estudiantes de la facultad de humanidades, se halló que el $81 \%$ de los evaluados se encuentran sexualmente activos, y el $78,5 \%$ de los sujetos de estudio utilizan al momento de la evaluación métodos anticonceptivos. En cuanto a los anticonceptivos empleados, un $46,3 \%$ de la muestra usa preservativos o condón, $16,5 \%$ pastillas o píldoras y 28 sujetos de los 121 evaluados, no recurre a ningún método anticonceptivo.

En lo referente a la actitud de los estudiantes hacia el método anticonceptivo utilizado, se observa de manera sobresaliente en la tabla 3 que lo emplean porque es fácil de conseguir, fácil de usar y no daña la salud con un $67,8 \%, 66,1 \%$ y $65,3 \%$ respectivamente.

Tabla 3. Opiniones sobre el uso del método anticonceptivo utilizado

\begin{tabular}{|l|c|c|c|c|c|c|c|}
\hline \multirow{2}{*}{$\begin{array}{l}\text { De acuerdo o } \\
\text { en desacuerdo }\end{array}$} & \multicolumn{2}{|c|}{ Es barato } & \multicolumn{2}{c|}{ No falla } & \multicolumn{2}{c|}{ Fácil de conseguir } & \\
\cline { 2 - 7 } & Frec. & $\%$ & Frec. & $\%$ & Frec. & $\%$ & \\
\hline Totalmente & 74 & $61,1 \%$ & 61 & $50,4 \%$ & 97 & $80,2 \%$ \\
\hline $\begin{array}{l}\text { Ni de acuerdo ni en } \\
\text { desacuerdo }\end{array}$ & 39 & $32,2 \%$ & 44 & $36,4 \%$ & 22 & $18,2 \%$ \\
\hline En desacuerdo & 8 & $6,6 \%$ & 16 & $13,2 \%$ & 2 & $1,6 \%$ \\
\hline Total & 121 & $100 \%$ & 121 & $100 \%$ & 121 & $100 \%$ \\
\hline $\begin{array}{l}\text { De acuerdo o en desacuerdo } \\
\text { Frec. }\end{array}$ & \multicolumn{2}{|c|}{ Fácil de usar } & No produce molestias & Produce mayor placer \\
\cline { 2 - 8 } & $\%$ & Frec. & $\%$ & Frec. & $\%$ & \\
\hline De acuerdo & 92 & $76 \%$ & 65 & $53,7 \%$ & 55 & $45,5 \%$ \\
\hline $\begin{array}{l}\text { Ni de acuerdo ni en } \\
\text { desacuerdo }\end{array}$ & 25 & $20,7 \%$ & 41 & $33,9 \%$ & 48 & $39,7 \%$ \\
\hline En desacuerdo & 4 & $3,3 \%$ & 15 & $12,4 \%$ & 18 & $14,8 \%$ \\
\hline Total & 121 & $100 \%$ & 121 & $100 \%$ & 121 & $100 \%$ \\
\hline
\end{tabular}


Continuación Tabla 3.

\begin{tabular}{|l|c|c|c|c|c|c|}
\hline \multirow{2}{*}{ De acuerdo o en desacuerdo } & \multicolumn{2}{|c|}{$\begin{array}{c}\text { No está en contra de mi } \\
\text { religión }\end{array}$} & $\begin{array}{c}\text { Produce control sobre mi } \\
\text { cuerpo }\end{array}$ & \multicolumn{2}{c|}{ No daña la salud } \\
\hline & Frec. & $\%$ & Frec. & $\%$ & Frec. & $\%$ \\
\hline De acuerdo & 53 & $44,6 \%$ & 43 & $35,5 \%$ & 86 & $71,1 \%$ \\
\hline $\begin{array}{l}\text { Ni de acuerdo ni en } \\
\text { desacuerdo }\end{array}$ & 60 & $49,6 \%$ & 65 & $53,7 \%$ & 29 & $24 \%$ \\
\hline En desacuerdo & 7 & $5,8 \%$ & 13 & $10,8 \%$ & 6 & $4,9 \%$ \\
\hline Total & 121 & $100 \%$ & 121 & $100 \%$ & 121 & $100 \%$ \\
\hline
\end{tabular}

Fuente: elaboración propia.

Tabla 4. Opiniones hacia cada uno de los métodos anticonceptivos

\begin{tabular}{|c|c|c|c|c|c|c|}
\hline & \multicolumn{2}{|c|}{ Hormonales } & \multicolumn{2}{|c|}{ Métodos de barrera } & \multicolumn{2}{|c|}{ Dispositivos intrauterinos } \\
\hline Opiniones & Frec. & $\%$ & Frec. & $\%$ & Frec. & $\%$ \\
\hline Mala opinión & 24 & $21,3 \%$ & 6 & $5 \%$ & 25 & $20,6 \%$ \\
\hline Ni mala ni buena & 32 & $25,3 \%$ & 30 & $24,8 \%$ & 23 & $19 \%$ \\
\hline Buena opinión & 42 & $34,5 \%$ & 84 & $69,4 \%$ & 36 & $29,7 \%$ \\
\hline No lo conozco & 23 & $18.8 \%$ & 1 & $0,8 \%$ & 37 & $30,6 \%$ \\
\hline \multirow[t]{2}{*}{ Total } & 121 & $100 \%$ & 121 & $100 \%$ & 121 & $100 \%$ \\
\hline & \multicolumn{2}{|c|}{ Irreversibles } & \multicolumn{2}{|c|}{ Ritmo } & \multicolumn{2}{|c|}{$\begin{array}{c}\text { Anticonceptivos de } \\
\text { emergencia }\end{array}$} \\
\hline Opiniones & Frec. & $\%$ & Frec. & $\%$ & Frec. & $\%$ \\
\hline Mala opinión & 23 & $19 \%$ & 61 & $50,4 \%$ & 61 & $50,4 \%$ \\
\hline Ni mala ni buena & 33 & $27 \%$ & 30 & $24,8 \%$ & 25 & $20,7 \%$ \\
\hline Buena opinión & 56 & $46 \%$ & 24 & $19,8 \%$ & 23 & $6,6 \%$ \\
\hline No lo conozco & 9 & $8 \%$ & 6 & $5 \%$ & 12 & $9,9 \%$ \\
\hline \multirow[t]{2}{*}{ Total } & 121 & $100 \%$ & 121 & $100 \%$ & 121 & $100 \%$ \\
\hline & \multicolumn{2}{|c|}{ Abstinencia } & & & & \\
\hline Opiniones & Frec. & $\%$ & & & & \\
\hline Mala opinión & 59 & $48,8 \%$ & & & & \\
\hline Ni mala ni buena & 23 & $19 \%$ & & & & \\
\hline Buena opinión & 36 & $29,7 \%$ & & & & \\
\hline No lo conozco & 3 & $2,5 \%$ & & & & \\
\hline Total & 121 & $100 \%$ & & & & \\
\hline
\end{tabular}

Fuente: elaboración propia. 
De acuerdo con los resultados obtenidos en la tabla 4, los métodos sobre los cuales los estudiantes tienen opiniones negativas en mayor proporción son la abstinencia, el ritmo y las pastillas de emergencia $(48,8 \%, 50,4 \%$ y $50,4 \%$ respectivamente), no siendo este último considerado un método de planificación familiar, mientras que sobre los preservativos $(69,4 \%)$, inyecciones $(47,2 \%)$, vasectomía $(45,5 \%)$, pastillas $(44,6 \%)$ y ligaduras de trompas $(43 \%)$, los estudiantes poseen opiniones favorables. Los métodos anticonceptivos sobre los cuales los sujetos poseen menor o nulo conocimiento son los óvulos, dispositivo intrauterino y los implantes (33,9\%, 30,6\% y $26,4 \%$ respectivamente).

Tabla 5. Opiniones generales sobre métodos anticonceptivos y género

\begin{tabular}{|c|c|c|c|c|c|c|c|c|c|}
\hline \multirow{2}{*}{ Opiniones } & \multicolumn{2}{|c|}{ Femenino } & \multicolumn{2}{|c|}{ Masculino } & \multicolumn{2}{|c|}{ Total } & \multirow[t]{2}{*}{ Chi2 } & \multirow[t]{2}{*}{ Gl. } & \multirow[t]{2}{*}{ Sig. } \\
\hline & Frec. & $\%$ & Frec. & $\%$ & Frec. & $\%$ & & & \\
\hline Muy mala & 1 & $0,8 \%$ & 4 & $3,3 \%$ & 5 & $4,1 \%$ & \multirow{7}{*}{$9,500^{a}$} & \multirow{7}{*}{5} & \multirow{7}{*}{,091 } \\
\hline Algo mala & 7 & $5,8 \%$ & 1 & $0,8 \%$ & 8 & $6,6 \%$ & & & \\
\hline Ni mala ni buena & 18 & $14,9 \%$ & 13 & $10,7 \%$ & 31 & $25,6 \%$ & & & \\
\hline Algo Buena & 41 & $33.9 \%$ & 23 & $19 \%$ & 64 & $52,9 \%$ & & & \\
\hline Muy buena & 6 & $5 \%$ & 5 & $4,1 \%$ & 11 & $9,1 \%$ & & & \\
\hline No lo conozco & 0 & $0 \%$ & 2 & $1,7 \%$ & 2 & $1,7 \%$ & & & \\
\hline Total & 73 & $60,3 \%$ & 48 & $39,7 \%$ & 121 & $100 \%$ & & & \\
\hline
\end{tabular}

Fuente: elaboración propia.

Según los resultados descritos en la tabla 5 , se logra constatar que no existe asociación entre el género y las opiniones generales de los estudiantes sobre métodos anticonceptivos $(0,091 \mathrm{p}>0,001)$. Es importante mencionar que más del $50 \%$ de los evaluados tienen una opinión favorable de los métodos anticonceptivos y el 25,6\% no tiene una opinión ni buena ni mala acerca de estos. Los estudiantes que mencionaron no conocer ningún método anticonceptivo $(1,7 \%)$ pertenecen al género masculino.

Por otra parte, al analizar las variables programa con opinión sobre métodos anticonceptivos se observa que no existe asociación entre las misma. $(0,517 \mathrm{p}>0,001)$. Es importante mencionar que más del $10,7 \%$ de los estudiantes evaluados tienen una opinión poco favorable de los métodos anticonceptivos y el 25,6\% no tiene una opinión ni buena ni mala acerca de estos presentándose en mayor proporción en estudiantes de derecho. Los estudiantes que mencionaron no conocer ningún método anticonceptivo $(1,7 \%)$ pertenecen al programa de cine y audiovisuales y antropología.

\section{DISCUSIÓN}

Inicialmente, la investigación buscó conocer las opiniones de los estudiantes de la Facultad de Humanidades de la Universidad del Magdalena frente a las conductas relacionadas con la salud reproductiva, encontrando en los resultados que el $81 \%$ los estudiantes se encuentran sexualmente activos, de los cuales en gran medida utilizan algún método anticonceptivo, mostrando preferencias por el uso de métodos reversibles de barrera y hormonales (preservativo y pastillas o píldoras) lo cual se corrobora con otras investigaciones ${ }^{1721-28}$, así mismo, el $3 \%$ de los evaluados que se encuentran sexualmente activos, no emplean ningún tipo de método de anticoncepción y pertenecen al género masculino, estos resultados contrastan con otras investigaciones en las que se resalta que un alto índice de los sujetos que participaron en este estudio no planifican $(49 \%)^{27}$.

Con relación a los resultados sobre las opiniones que los estudiantes tienen acerca de los métodos de 31 anticoncepción, se encontró que los evaluados presentan 
en mayor proporción opiniones negativas (malas y muy malas) sobre la abstinencia, el ritmo y las pastillas de emergencia ${ }^{29}$, lo cual se opone a otros hallazgos en los que se plantea que los evaluados consideran a los anticonceptivos de emergencia como un método contraceptivo más ${ }^{30}$. Por su parte los sujetos de estudio presentan opiniones positivas sobre los preservativos, inyecciones, vasectomía, pastillas y ligaduras de trompas ${ }^{28,29}$. No obstante expresan no tener conocimiento de métodos como los óvulos, dispositivo intrauterino, implantes, lo cual se contrapone a lo planteado por investigaciones que destacan el amplio conocimiento de la muestra sobre métodos como el preservativo y el DIU en segundo lugarit ${ }^{16}$.

Es importante resaltar la tendencia que muestran los estudiantes a recurrir a métodos anticonceptivos sobre los cuales tienen una opinión positiva y se encuentran a un bajo costo economico ${ }^{26,31-33}$, evidenciando opiniones favorables en primer lugar sobre los métodos de barrera y en segundo lugar sobre los hormonales, los cuales son los más usados por los sujetos de investigación. Lo anterior contrasta con lo expuesto por otros estudios, en los que se concluye que el conocimiento que los evaluados poseen sobre los anticonceptivos no se relaciona con su uso, ya que existe un alto porcentaje de sujetos sexualmente activos sin cuidados anticonceptivos ${ }^{27}$.

Al momento de establecer la relación entre el género y las opiniones de los estudiantes sobre los métodos anticonceptivos se determinó que no existe asociación significativa entre estas dos variables, lo que indica que las opiniones no dependen del hecho de ser mujer $\mathrm{u}$ hombre ${ }^{24}$. Aunque los hallazgos destacan que no existe una asociación entre el género y las opiniones, se hace necesario resaltar que los estudiantes que mencionaron no conocer ningún método anticonceptivo pertenecen al género masculino. Asimismo, no existe asociación entre el programa y las opiniones de los estudiantes sobre métodos anticonceptivos. Los estudiantes que mencionaron no conocer ningún método anticonceptivo pertenecen al programa de antropología, cine y audiovisuales.

De acuerdo con los resultados de este estudio se puede 32 plantear a manera de conclusión que:

Los estudiantes que se encontraban sexualmente activos al momento de la evaluación utilizan en mayor proporción algún método anticonceptivo, mostrando preferencias por el uso del preservativo y de las píldoras. El 3\% de los evaluados que se encuentran sexualmente activos, no emplean ningún tipo de método de anticoncepción y pertenecen al género masculino.

Los evaluados presentan en mayor proporción opiniones negativas (malas y muy malas) sobre la abstinencia, el ritmo y las pastillas de emergencia, mientras se observan opiniones positivas sobre los preservativos, inyecciones, vasectomía, pastillas y ligaduras de trompas.

Un alto porcentaje de los estudiantes expresan no tener conocimiento sobre los óvulos, dispositivo intrauterino y los implantes como métodos anticonceptivos.

Al momento de establecer la relación entre el género y las opiniones de los estudiantes sobre los métodos anticonceptivos se determinó que no existe asociación significativa entre estas dos variables, lo que indica que las opiniones no dependen del hecho de ser mujer u hombre. Si bien los resultados resaltan que no existe una asociación entre el género y las opiniones, se hace necesario destacar que los estudiantes que mencionaron no conocer ningún método anticonceptivo pertenecen al género masculino.

No existe asociación entre el programa y las opiniones de los estudiantes sobre métodos anticonceptivos. Los estudiantes que mencionaron no conocer ningún método anticonceptivo pertenecen al programa de cine y audiovisuales y antropología.

Los hallazgos de este estudio sugieren la importancia de realizar estudios diagnósticos a nivel general sobre la vulnerabilidad de la población estudiantil de padecer una ETS o de embarazos no planeados a fin de plantear estrategias educativas de promoción y prevención que permitan reducir los altos índices de estas problemáticas que se presentan a nivel mundial.

\section{DECLARACIÓN SOBRE CONFLICTOS DE INTERESES}

Los autores no reportan conflictos a declarar relacionados con la investigación.

\section{REFERENCIAS BIBLIOGRÁFICAS}

1. Organización Mundial de la Salud. Estrategia mundial de prevención y control de las infecciones de transmisión sexual 2006-2015. Informe de un grupo científico de la OMS. Ginebra: OMS; 2007. 
2. Organización Panamericana de la Salud. Estrategia y plan de acción regional sobre los adolescentes y jóvenes 2010 - 2018. Informe de un grupo científico de la OPS. Washington. OPS; 2010.

3. Ministerio de la Protección Social. Política nacional de salud sexual y reproductiva. Informe de un grupo científico del Ministerio de la Protección social. Bogotá D.C. Ministerio de la Protección Social; 2003.

4. Alvis N, Mattar S, García J, Conde E, Díaz A. Infecciones de Transmisión Sexual en un Grupo de Alto Riesgo de la Ciudad de Montería, Colombia. Rev. salud pública. 2007; 9 (1):86-96.

5. Ministerio de Salud y Protección Social. Plan nacional para la atención del cáncer en Colombia 2012 - 2020. Informe de un grupo de científicos del Ministerio de Salud y Protección Social. Bogotá D.C: Ministerio de Salud y Protección Social; 2012.

6. United Nations Population Information Network-POPIN. Informe de la Conferencia Internacional sobre la Población y el Desarrollo. El Cairo: 5 a 13 de septiembre de 1994); 1994

7. Organización Mundial de la Salud. Infecciones de transmisión sexual y otras infecciones del tracto reproductivo: una guía para la práctica básica. Informe de un grupo científico de la OMS. Ginebra. Organización Mundial de la Salud; 2015.

8. Vinaccia S, Quiceno JM, Gaviria AM, Soto AM, Gil MD, Ballester R. Conductas Sexuales de Riesgo para la Infección por VIH/SIDA en Adolescentes Colombianos. Terapia Psicológica. 2007;25(1):39-50.

9. Arrivillaga M, Correa D, Tovar LM, Zapata H, Varela MT, Hoyos, PA. Infecciones de transmisión sexual en la región Pacífica colombiana: implicaciones para población en situación de vulnerabilidad étnica, social y económica. Pensamiento Psicológico. 2011;9(16):145-52.

10. Fundación Mexicana para la Salud A.C. Guía de prevención, diagnóstico y tratamiento de las ITS. Dirigida a personal de servicios de salud. Informe de un grupo científico de la Fundación Mexicana para la Salud A.C. México. D.F: Fundación Mexicana para la Salud A.C; 2011.

11. Salvo A. Infecciones de Transmisión Sexual (ITS) en Chile. REV. MED. CLIN. CONDES. 2011;22(6):813-24.

12. Villaseñor A, Caballero R, Hidalgo A, Santos JI. Conocimiento objetivo y subjetivo sobre el VIH/SIDA como predictor del uso de condón en adolescentes. Salud Publica Mex. 2003;45(1):73-80.

13. Vera LM, López N, Ariza ND, Díaz LM, Flórez Y, Franco SG, et al. Asociación entre el área de estudio y los conocimientos y comportamientos frente a la transmisión del VIH/SIDA en los estudiantes de la Universidad Industrial de Santander. Colombia Médica. 2004;35(2):62-8.
14. Vásquez ML, Argote LA, Castillo E, Mejía ME,Villaquirán ME. La educación y el ejercicio responsable de la sexualidad en adolescentes. Colombia Médica. 2005;36(2):33-42.

15. Flores F, Leyva R. Representación social del SIDA en estudiantes de la Ciudad de México. Salud PublicaMex. 2003;45(5):624-31.

16. Santín C, Torrico E, López MJ, Revilla C. Conocimiento y |ción de los métodos anticonceptivos y su relación con la prevención de enfermedades de transmisión sexual en jóvenes. Anales de psicología. 2003;19(1):81-90.

17. González FG. Conocimientos, actitudes y prácticas en salud sexual y reproductiva en jóvenes entre 14 y 25 años de edad de estratos 1 y 2 del SISBEN de un municipio del Departamento de Cundinamarca. Acta Colombiana de Psicología. 2004;12:59-68.

18. Pérez C, Pick S. Conducta Sexual Protegida en Adolescentes Mexicanos. Revista Interamericana de Psicología/Interamerican Journal of Psychology. 2006;40(3):333-340.

19. Profamilia. Encuesta Nacional de Demografía y Salud. Informe de un grupo de científicos de Profamilia. Bogota: Profamilia; 2010

20. Behar, DS. Metodología de la investigación. Behar, DS. Cali: Editorial Shalom; 2008 [citado 2015 Nov 21]. Disponible en: http://museoarqueologico.univalle.edu.co/imagenes/ Proyecto \% 20de \%20Grado\% 201/lecturas/Libro \% 20 metodologia \% 20investigacion. \%20Libro\%20NB.pdf

21. Santoyo S, Ponce ER, Gómez FJ, Fernández MA, Domínguez J, Díaz V. Confiabilidad y validez de constructo de una escala para evaluar las actitudes y opiniones hacia las prácticas anticonceptivas en 14 países de América Latina. Revista de la Facultad de Medicina de la UNAM 2010;53(4):15-22.

22. Ministerio de Salud. Título II: De la investigación en seres humanos; 1993 [citado 2016 May 11]. Disponible en: web: http://www.dib.unal.edu.co/promocion/ etica_res_8430_1993.pdf

23. Ministerio de la Protección Social. Ley 1090 del 2006; 2006 [citado 2016 May 11]. Disponible en: http://www.upb.edu.co/pls/portal/docs/PAGE/ GPV2_UPB_MEDELLIN/PGV2_M030_PREGRADOS/ PGV2_M030040020_PSICOLOGIA/CODIGO_ETICO/ CODIGO\%20DEONTOLOGICO \%20Y\%20BIOETICO.PDF

24. Hernando V, Álvarez M, Arriola L, Arroyo S, Ballester E, Bermúdez P, et al. Conocimientos y uso de anticonceptivos en la población inmigrante latinoamericana en la Comunidad Autónoma de Madrid. Boletín epidemiológico semanal. 2005;13(4):37-48.

25. González E, Molina T, Montero A, Martínez V, Leyton C. Comportamientos sexuales y diferencias de género en adolescentes usuarios de un sistema público de salud universitario. Rev. méd. Chile. 2007; 135(10): 1261-69. 
26. Gómez PI, Arguello K, Cáceres C, Gutiérrez E, Rodríguez A, Rojas L, et al. Anticoncepción de emergencia hormonal: conocimientos, actitudes y prácticas en estudiantes universitarias. Estudio de corte transversal. Colombia. Revista Colombiana de Enfermería. 2008;5 (5): 9-14.

27. Castañeda O, Ortega NG, Reyes Y, Segura O, Morón L. Conocimientos, actitudes y prácticas en salud sexual y reproductiva, en Yopal, Casanare. Investigaciones Andinas. 2009; 11 (19): 31-48.

28. Castro M, Peniche AC, Peña JL. Conocimientos, mitos y practicas sobre el uso de métodos anticonceptivo asociados al incremento de embarazos no planificados en estudiantes adolescentes en dos instituciones educativas en la ciudad de Cartagena. Tesis de grado.[Internet]. 2012 [citado 2015 Nov 21]. Disponible en: http:// siacurn.curnvirtual.edu.co:8080/xmlui/bitstream/ handle/123456789/577/CONOCIMIENTOS, $\% 20$ MITOS \% 20Y \% 20PRACTICAS \% 20SOBRE \% 20 EL \% 20USO \% 20DE \% 20M \% C3 \% 89TODOS \% 20 ANTICONCEPTIVO.pdf? sequence $=1$
29. Mendoza LA, Arias M, Pedroza M, Micolta P, Ramírez A, Cáceres $\mathrm{C}$, et al. Actividad sexual en adolescencia temprana: problema de salud pública en una ciudad colombiana. Revista Chilena de Obstetricia Ginecología. 2012; 77(4):271-279.

30. Rengifo HA, Córdoba A, Serrano M. Conocimientos y prácticas en salud sexual y reproductiva de adolescentes escolares en un municipio colombiano. Rev. salud pública. 2012; 14 (4): 558-569.

31. Monterrosa A, Ulloque L, Carriazo S. Calidad del dormir, insomnio y rendimiento académico en Estudiantes de Medicina. Duazary. 2014 Dic; 11 (2): 85-97

32. Ferrel F, Ortiz A, Forero L, Herrera M, Peña Y. Estilos de vida y factores sociodemográficos asociados en adolescentes escolarizados del Distrito de Santa Marta, Colombia. Duazary. 2014 Dic; 11 (2): 105-14

33. Pedraza L, Socarrás X, Sevilla R, López J, Jiménez J. Opiniones hacia las prácticas anticonceptivas en estudiantes de la Universidad del Magdalena, Santa Marta (Colombia). Revista Ciencia y Salud. 2014; 6 (2): 82-91.

Para citar este artículo: Rodríguez-De Ávila U, Bocanegra-Sandoval J, Maestre-Oñate E, Lozano-González S. Opiniones sobre las prácticas anticonceptivas en estudiantes de una Facultad de Humanidades en Santa Marta. Duazary. 2017 enero; 14 (1): 25 - 34 